\title{
The Impact of Nursing Education on Cardiac Patient's Knowledge Regarding their Lifestyle in Tertiary Care Hospital Peshawar KP
}

\author{
Muslim Shah" ${ }^{1 *}$, Adnan Khan', Zeeshan Khan', Sania Amir ${ }^{2}$ and Farah $\mathrm{Naz}^{2}$ \\ ${ }^{1}$ Assistant Professor, Rehman College of Nursing, Rehman Medical Institute, Peshawar, Pakistan \\ ${ }^{2} B S N 4^{\text {th }}$ Year Students, Rehman College of Nursing, Rehman Medical Institute, Peshawar, Pakistan \\ *Corresponding author: Muslim Shah, Assistant Professor, Rehman College of Nursing, Rehman Medical Institute, Peshawar, \\ Pakistan
}

\begin{abstract}
Objectives: The objective of the study was to assess the impact of nursing education on cardiac patient knowledge regarding their lifestyle.

Methodology: Quasi-experimental study was conducted in private tertiary care hospital. A well-structured questionnaire was adopted and explained to every participant during data collection. Data was collected through convenient sampling. Data analysis was done by SPSS version.

Result: Out of 39 participants, 4(10.3\%) participants fell under excellent category, 19(48.7\%) had good knowledge, and $16(41.0 \%)$ had poor knowledge before educational intervention. After the intervention, 38(97.4\%) participants fell under excellent category and $1(2.6 \%)$ had good knowledge. The mean score was 20.85 with a standard deviation of 7.084 before session; while, the mean score after session was 33.69 with a standard deviation of 2.430. Furthermore, paired T-test was applied to evaluate the effectiveness of education. The result was highly significant with a p-value of 0.000 .
\end{abstract}

Conclusion: The findings of this study support the effectiveness of cardiac education that shows an increase in patient's knowledge and change in behavior. Therefore, educational program is significant for cardiac patients.

\section{Background}

Heart disease or cardiovascular diseases(CVD) is a broad term used to describe various diseases like Coronary Artery Disease (CAD), Myocardial Infarction, Angina, Atrial Fibrillation, Heart Rhythm Disorders, Cardiomyopathy and Heart Failure, and other disorders of heart. According to the Centers for Disease Control and Prevention, Coronary Artery Disease is the most common type of heart disease and could lead to heart attacks. Different nations have been developing policies to condense the risk of heart disease in their populations Yee Chow, Chan [1] Cardiac or Cardiovascular (heart \& vessels) diseases (CVDs) are the leading cause of death. It has been observed that $33 \%$ of total deaths in 2011 are due to cardiac problems. The risk factors which lead to cardiac diseases are gender being male, increasing age, family history, high cholesterol, smoking, diabetes, hypertension, obesity, psychosocial factors, and alcohol consumption, low intake of fruit and vegetables, and physical inactivity. Providing adequate knowledge about coronary artery disease (CAD) through cardiac educational program is believed to be an important requisite for adherence to healthy lifestyle Tawalbeh \& Ahmad [2], Chow [1] conducted a cross sectional sample survey with the purpose of assessing cardiac knowledge and cardiac misconception. The result of the study highlights that the cardiac knowledge test was 12.27 out of 18(SD 2.38), with a range of 2-17. For cardiac misconception, the mean score was 6.98 out of 20 (SD 2.84), with a range of $0-14$. The chi-square test found some association between experience of caring for cardiac patient and misconception about stress and physiology. Another Quasi experimental study carried out by Ross, Ohlsson, Blomberg, and 
Gustafsson [3], to assess and individualize patient education at a nurse led heart failure clinic. The results of the study showed that there was no difference between control and intervention group. However, intervention group had more information as compared to control group concerning their personal situation.

Similarly, a study was conducted by Ghisi et al. [3] to investigate the impact of education on cardiac patient knowledge. In general, the result of the study showed (57\%) studies as fair and 5\% studies as poor in quality.

Moreover, a quasi-experimental study was conducted by Siraje et al. [8]. The results showed that self-care behaviors in patients with heart failure were influenced by awareness, and their practice improved with increasing their knowledge. Therefore, it is recommended to give an educational pamphlet or video to patients with heart failure. Likewise, the study of Kadda, Marvaki, and Panagiotakos shows the role of nursing education after a cardiac event. Nursing education in cardiac rehabilitation can increase health outcomes and reduce the risk of a new cardiac event. A health educational program prepared by nurses for patients after a cardiac event or surgery improves patients' knowledge of their illness and awareness of behavioral changes to prevent a new event or readmission to hospital. The purpose of this study was to evaluate the impact of nursing education on cardiac patient's knowledge regarding their lifestyle.

Research Question: Is there any impact of nursing education on cardiac patient's knowledge regarding their lifestyle?

Hypothesis: Null hypothesis: There is no association between nursing education and patient's knowledge regarding their lifestyle.

Alternate Hypothesis: There is an association between nursing education and patient's knowledge regarding their lifestyle.

\section{Methodology}

Research Design: The quasi-experimental study design was used in this study. This particular research design was used because it normally allows the researcher to control the assignment as per treatment condition Dinardo [5].

\section{Population and Study Setting}

Population of the study was admitted cardiac patients at private tertiary care hospital Peshawar.

\section{Sample and Sampling Technique}

Sample size was calculated by using Rao soft software, with the population of 80 with $5 \%$ margin of error and $5 \%$ non-responsive rate. The calculated sample size was 39. A convenient sampling technique was used for sample selection because of accessibility to the patient.

\section{Data Collection Tool}

Questionnaire was adopted and modified for data collection after permission from the author. The questionnaire consisted of 12 questions about cardiac diseases and life style of cardiac patients.

\section{Data Analysis}

Statistical package for social sciences (SPSS) version 22 was used for data analysis. Frequencies were calculated for nominal and ordinal data, while mean and standard deviation was calculated for continuous variables. Paired T-test was applied to identify association among variables. Criteria were developed for data analysis in which patients were divided into categories on the basis of pre and post education knowledge. The total score was 36 , in which $>30=$ excellent, $21-29=$ good and $<20$ was categorized as poor.

\section{Ethical Consideration}

Approval from Chief Nursing Officer (CNO) was taken for the data collection. An informed consent form was distributed and explained to each participant. Anonymity was guaranteed to all the participants and confidentiality was maintained throughout the study. Inclusion criteria

a) Subjects that are mentally competent and oriented

b) Admitted patient to cardiac wards for 24 hours

c) Age 18 years or above

d) Subjects receiving nursing care

\section{Results}

The study consisted of 39 participants; 23 (59\%) males and 16 (41\%) females as shown in Figure 1. Age of the participants was 20 to $>60$ years. From $20-40=9(23.1 \%)$, from $40-60=19(48.7 \%)$ and $>60=11(28.2 \%)$ shown in Figure 2. The entire participants were married. $21(53.8 \%)$ participants had $<1$ year of duration of disease while $18(46.2 \%)$ had $>1$ year of duration of disease. $20(51.3 \%)$ participants had family history of the disease while $19(48.7 \%)$ had no family history of the disease. Moreover, mean and standard deviation was calculated to see knowledge of the participants regarding their lifestyle before and after education. Before education, the mean score was 20.85 with a standard deviation of 7.084; while, the mean score after education was 33.69 with a standard deviation of 2.430 . Besides, the participants were divided into categories on the basis of pre education knowledge and post education knowledge. The total score was 36, in which $>30=$ excellent, 21-29=good and $<20$ was categorized as poor. Before educational intervention, $4(10.3 \%)$ participants fell under excellent category, 19(48.7\%) had good knowledge and 16(41.0\%) had poor knowledge as shown in Figure 3. After the education session, 38(97.4\%) participants fell under the excellent category and $1(2.6 \%)$ had good knowledge shown in Figure 4. Furthermore, paired T-test was applied to evaluate the effectiveness of education. The result was highly significant with a p-value of 0.000 . Hence the null hypothesis was rejected, and it has been proved that there is association between nursing education and cardiac patient knowledge regarding their lifestyle. 


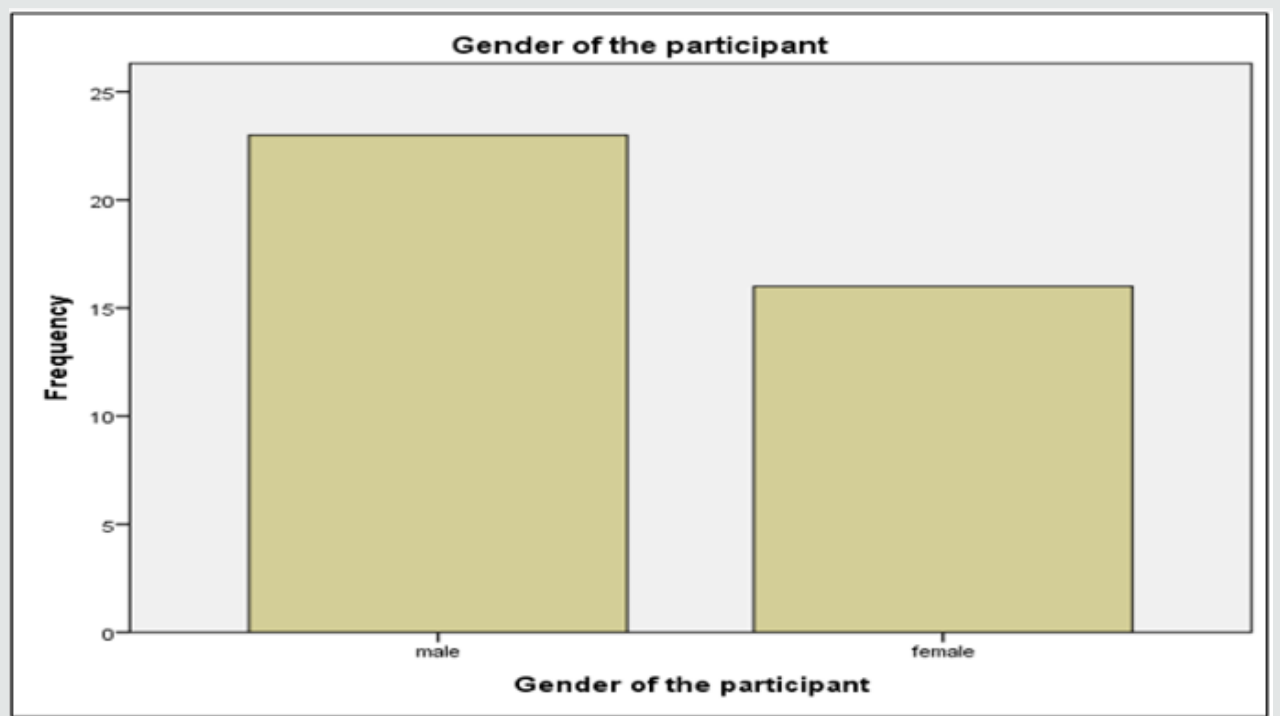

Figure 1.

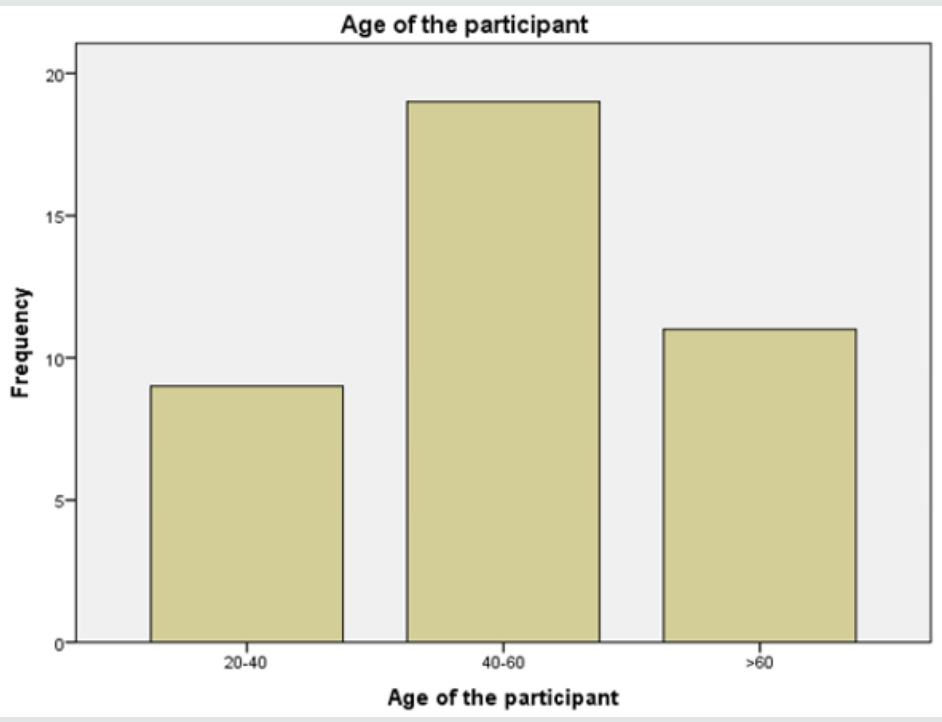

Figure 2.

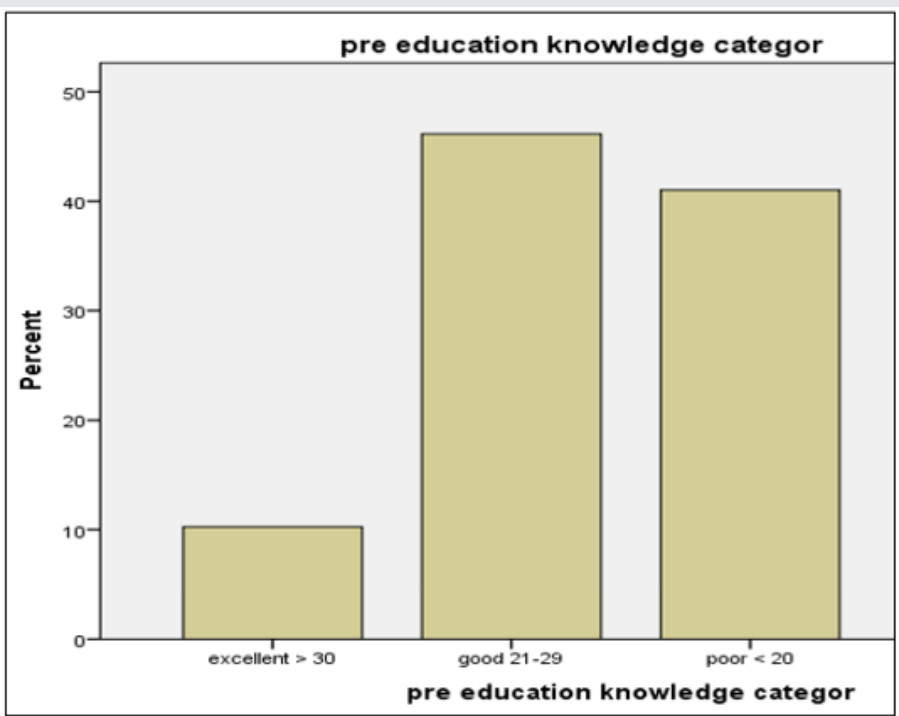

Figure 3. 


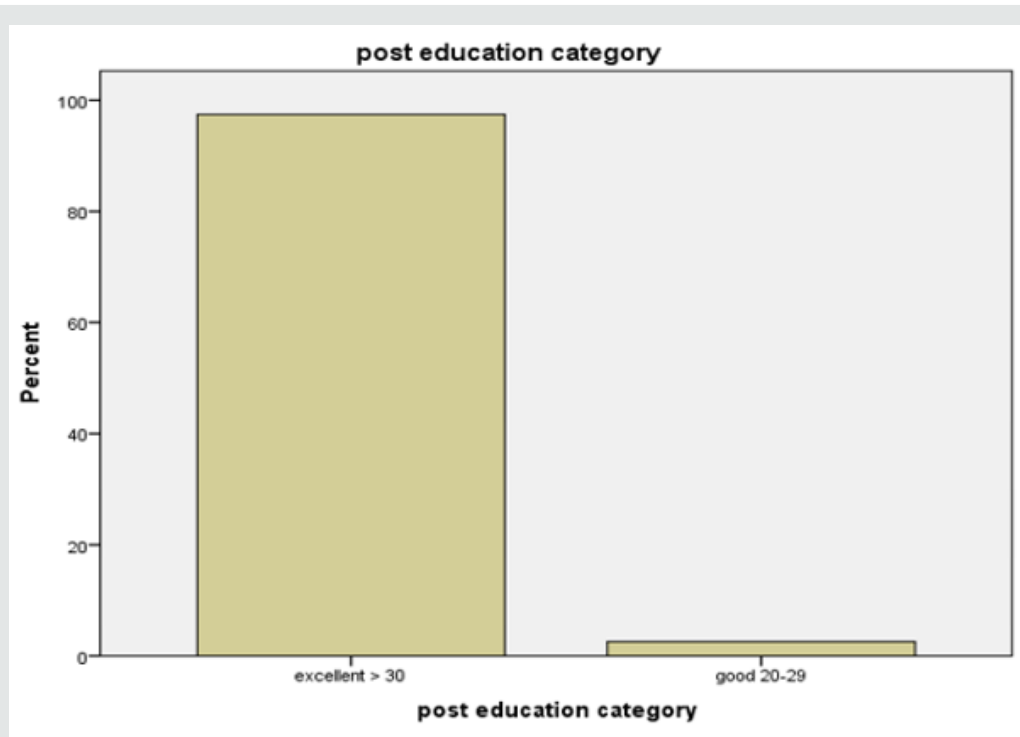

Figure 4 .

\section{Discussion}

The results of this study are similar to study conducted by Ghisi et al. [4] which shows that education regarding lifestyle of the cardiac patient is significant because it results in increase of patients' knowledge, and it may also change patients' behavior. A study by Winkler (2014) identified that 57 patients, completing pre and post-test knowledge, had an increase in knowledge of cardiovascular diseases $(\mathrm{p}<0.001)$; similar results were obtained from the current study $(\mathrm{p}<0.0001)$. The results of the current study also showed that educational intervention enhances the knowledge of patient with cardiac diseases. The mean score of the study was 33.69(SD 2.430) after education with a highly significant p-value of 0.0001 , which is consistent with the results of the study carried by Tawalbeh and Ahmad [2]. Another study by Siraje et al. [6]. showed that self-care behaviors in patients with heart diseases were influenced by awareness, and their practice improved with increasing their knowledge which is parallel with results of this study. Besides, this study recommended daily self-care activities for people living with heart failure including weight monitoring, adherence to a strict medication and diet, self-monitoring of symptoms and regular exercise which is reliable with the study conducted by Baptiste et al. [7]. The study recommends that educating the patients about their lifestyle may affect their health in later life. Therefore, educating the patients about their lifestyle is very necessary in early phase of disease which can minimize the risk of further complication through changing the modifiable risk factors. Also, this study provide basis for conducting other studies which can address the gaps and limitations of this study $[8,9]$.

\section{Conclusion}

Most of the cardiac patients are unaware of their disease process, and they may also have inadequate knowledge regarding healthy lifestyle due to which their disease may get worse. Therefore, it is recommended to give education to patients regarding their lifestyle so that they can change their habits accordingly. The findings of this study also support the benefits of cardiac education which may bring an increase in patient's knowledge and behavior change.

\section{References}

1. Chow SKY, Chan YY, Ho SK, Ng KC (2017) Cardiac health knowledge and misconceptions among nursing students: implications for nursing curriculum design. BMC Nurs 16: 46.

2. Tawalbeh LI, Ahmad MM (2014) The effect of cardiac education on knowledge and adherence to healthy lifestyle. Clin Nurs Res 23(3): 245-258.

3. Ross A, Ohlsson U, Blomberg K, Gustafsson M (2015) Evaluation of an intervention to individualize patient education at a nurse-led heart failure clinic: a mixed-method study. J Clin Nurs 24(11-12): 1594-1602.

4. De Melo Ghisi G, Abdallah F, Grace SL, Thomas S, Oh P (2014) A systematic review of patient education in cardiac patients: do they increase knowledge and promote health behavior change?. Patient Educ Couns 95(2): 160-174.

5. Dinardo J (2008) Natural experiments and quasi-natural experiments. The New Palgrave Dictionary of Economics.

6. Seraji M, Tabatabaie P, Rakhshan F, Shahrakipour M (2013) The effect of educating self-care behaviors to patients with heart failure in hospitals of zahedan. Health Scope 2(2): 104-109.

7. Baptiste DL, Mark H, Groff Paris L, Taylor LA (2013) A nurse-guided patient-centered heart failure education program. Journal of Nursing Education and Practice 4(3).

8. Olga K (2012) The role of nursing education after a cardiac event. Health science journal 6(4): 634-646.

9. Ostrowski Winkler LA (2014) The effects of education on cardiovascular disease knowledge. 
(c) $\left(\begin{array}{l}\text { This work is licensed under Creative } \\ \text { Commons Attribution } 4.0 \text { License }\end{array}\right.$

To Submit Your Article Click Here:

Submit Article

DOI: $10.32474 /$ LOJNHC.2019.02.000133

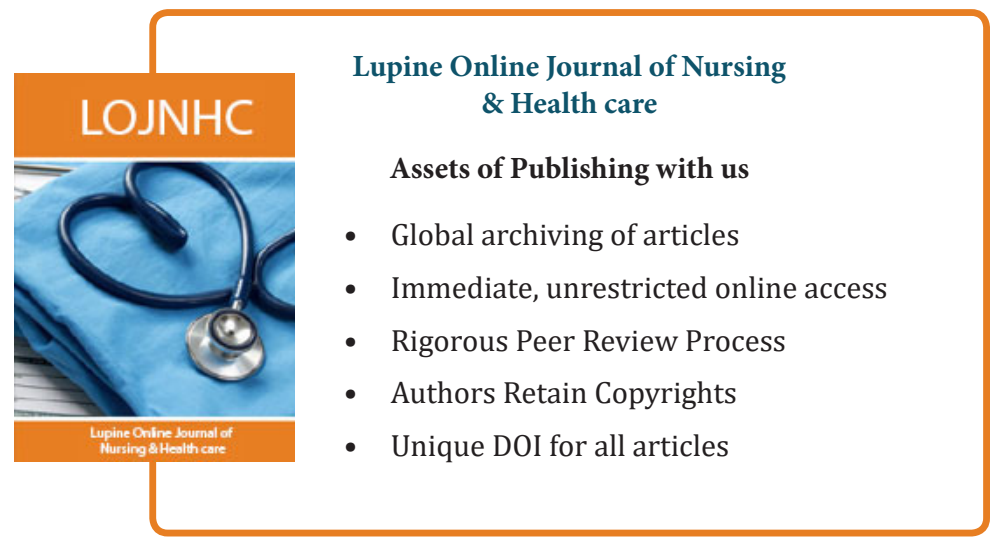

